\title{
Iterated sumsets and Hilbert functions
}

\author{
Shalom Eliahou* and Eshita Mazumdar ${ }^{\dagger}$
}

\begin{abstract}
Let $A$ be a finite subset of an abelian group $(G,+)$. For $h \in \mathbb{N}$, let $h A=A+\cdots+A$ denote the $h$-fold iterated sumset of $A$. If $|A| \geq 2$, understanding the behavior of the sequence of cardinalities $|h A|$ is a fundamental problem in additive combinatorics. For instance, if $|h A|$ is known, what can one say about $|(h-1) A|$ and $|(h+1) A|$ ? The current classical answer is given by

$$
|(h-1) A| \geq|h A|^{(h-1) / h},
$$

a consequence of Plünnecke's inequality based on graph theory. We tackle here this problem with a completely new approach, namely by invoking Macaulay's classical 1927 theorem on the growth of Hilbert functions of standard graded algebras. With it, we first obtain demonstrably strong bounds on $|h A|$ as $h$ grows. Then, using a recent condensed version of Macaulay's theorem, we derive the above Plünneckebased estimate and significantly improve it in the form
\end{abstract}

$$
|(h-1) A| \geq \theta(x, h)|h A|^{(h-1) / h}
$$

for $h \geq 2$ and some explicit factor $\theta(x, h)>1$, where $x \in \mathbb{R}$ satisfies $x \geq h$ and $|h A|=\left(\begin{array}{l}x \\ h\end{array}\right)$. Equivalently and more simply,

$$
|(h-1) A| \geq \frac{h}{x}|h A| .
$$

We show that $\theta(x, h)$ often exceeds 1.5 and even 2 , and asymptotically tends to $e \approx 2.718$ as $x$ grows and $h$ lies in a suitable range depending on $x$.

Keywords: Plünnecke's inequality; Standard Graded Algebra; Macaulay's Theorem; Binomial representation.

MSC2020: 11P70, 13P25, 05E40

${ }^{*}$ LMPA-ULCO, Calais, France. Email: eliahou(at)univ-littoral.fr

${ }^{\dagger}$ Stat-Math Unit, ISI Bengaluru. Email: eshita_vs(at)isibang.ac.in 


\section{Introduction}

Let $A$ be a nonempty finite subset of an abelian group $(G,+)$. For any $h \in \mathbb{N}_{+}=\{1,2, \ldots\}$, we denote by $h A$ the $h$-fold iterated sumset of $A$, i.e.

$$
h A=A+\cdots+A=\left\{x_{1}+\cdots+x_{h} \mid x_{i} \in A \text { for all } 1 \leq i \leq h\right\} .
$$

As usual, we set $0 A=\{0\}$. A central problem in additive combinatorics is to understand the behavior of $|h A|$ as $h$ grows. Asymptotically, it is known that $|h A|$ is eventually polynomial in $h$. See e.g. [6, 7, 11]. But not much is known about this polynomial and, for $h$ small, the behavior of $|h A|$ may wildly depend on the structure, or lack thereof, of $A$. For instance, if $A$ is a subset of $\mathbb{Z}$ such that $|A|=n$, then

$$
h(n-1)+1 \leq|h A| \leq\left(\begin{array}{c}
n-1+h \\
h
\end{array}\right)
$$

both bounds being attained in suitable cases: arithmetic progressions for the lower bound, and so-called $B_{h}$-sets for the upper bound. The latter is best understood by noting that this binomial coefficient counts the number of monomials of degree $h$ in $|A|$ commuting variables. See e.g. [18, Sections 2.1 and 4.5] or [4, Section 3.2].

Here we address the following question. If $h \geq 2$ and $|h A|$ is known, what estimates on $|(h-1) A|$ and $|(h+1) A|$ can one derive? The classical answer, given by Plünnecke's inequality and based on graph theory [13], is as follows:

$$
|(h-1) A| \geq|h A|^{(h-1) / h} .
$$

See also [16, 11, 18. In this paper, we derive this bound with a completely new approach, and we significantly improve it along the way. Our approach relies on Macaulay's classical 1927 theorem characterizing the Hilbert functions of standard graded algebras [9]. We apply that theorem to a suitable standard graded $K$-algebra $R=R(A)=\oplus_{h \geq 0} R_{h}$ having the property

$$
\operatorname{dim}_{K} R_{h}=|h A|
$$

for all $h \geq 0$. Using a recent condensed version of Macaulay's theorem [3], we improve (1) as follows. Denote

$$
\theta(x, h)=\frac{h}{x}\left(\begin{array}{l}
x \\
h
\end{array}\right)^{1 / h}
$$


for $x \in \mathbb{R}$ and $h \in \mathbb{N}$. If $|A| \geq 2$, our improved bound implies

$$
|(h-1) A| \geq \theta(x, h)|h A|^{(h-1) / h},
$$

where $x$ is the unique real number larger than $h$ such that $|h A|=\left(\begin{array}{l}x \\ h\end{array}\right)$. This ensures $\theta(x, h)>1$. In fact, the factor $\theta(x, h)$ often exceeds 1.5 and even 2 , as shown in Sections 5.2 and 5.3. For instance, for $h=12$ we have $\theta(x, 12))>2.013$ for all $x \geq 50$. This implies in turn that if $A$ satisfies $|12 A| \geq 121,400,000,000$, then

$$
|11 A| \geq 2|12 A|^{11 / 12} .
$$

The wide occurrence of the case $\theta(x, h) \geq 2$ is described in more detail in Section 5.3. Remarkably, for $x$ large enough and suitable values of $h$ depending on $x$, the factor $\theta(x, h)$ approaches $e \approx 2.718$, the basis of the natural logarithm. For instance, this occurs for all $x \geq 10^{6}$ at $h=3000$. See also Section 5.4, where strong evidence suggests that $\lim _{x \rightarrow \infty} \theta\left(x,\left\lfloor x^{1 / 2}\right\rfloor\right)=e$. Three general remarks are in order here.

Remark 1.1. Our results are stated for finite subsets of an abelian group $G$, but they hold more generally if $G$ is a commutative semigroup, as in [12] for instance.

Remark 1.2. Commutative algebra has already been applied to estimate the growth of iterated sumsets. In particular, the Hilbert polynomial of graded modules has been used to determine the asymptotic behavior of the function $h \mapsto|h A|$, and more generally of the function $\left(h_{1}, \ldots, h_{r}\right) \mapsto$ $\left|B+h_{1} A_{1}+\cdots+h_{r} A_{r}\right|$. See [6, 7, 12, 11]. However, to the best of our knowledge, the only previous application of Macaulay's theorem to additive combinatorics is in [3], where the above-mentioned condensed version is established and applied to yield an asymptotic solution of Wilf's conjecture on numerical semigroups.

Remark 1.3. Another way of comparing $|h A|$ with $|(h-1) A|$ has been made, at least for $A \subset \mathbb{Z}$, by seeking to bound the difference $|h A|-|(h-1) A|$ from below rather than the quotient $|h A| /|(h-1) A|$ from above [8]. In the study of the difference $|h A|-|(h-1) A|$, a main tool is Kneser's theorem, whereas for the quotient $|h A| /|(h-1) A|$, the classical one is Plünnecke's inequality, and an additional one is now Macaulay's theorem as made plain in this paper. 
There is a vast literature on Plünnecke's inequality, its rich applications to additive combinatorics and its successive refinements, such as the PlünneckeRuzsa inequality for instance [17]. Besides dedicated chapters in [16, 11, 18], see also the nice survey [14] and its many references.

The contents of this paper are as follows. In Section 2, we construct a graded algebra $R(A)$ whose Hilbert function exactly models the sequence $|h A|_{h \geq 0}$. In Section 3, we recall Macaulay's theorem on Hilbert functions and the recent condensed version that we shall use. We prove our main results in Section 4. The first ones, Theorems 4.3 and 4.4, are obtained by applying Macaulay's theorem and its condensed version to the algebra $R(A)$. The strength of these results is then illustrated with the specific case $|5 A|=100$. Here, Plünnecke's inequality implies $|4 A| \geq 40$ and $|6 A| \leq 251$, whereas our method yields much sharper and almost optimal bounds, namely $|4 A| \geq 61$ and $|6 A| \leq 152$. As our next main result, Theorem 4.9, we derive the Plünnecke-based estimate (1) from Theorem 4.4 and improve it by some multiplicative factor $\theta(x, h)>1$. The numerical behavior of that factor is studied in Section 5 and shown to often exceed 1.5 and even 2. In Section 6 , we give a presentation of $R(A)$ by generators and relations. We conclude the paper in Section 7 with related questions and remarks.

\section{The graded algebra $R(A)$}

Let $A$ be a finite subset of an abelian group. Here we associate to $A$ a standard graded algebra $R(A)$ whose Hilbert function models the sequence $|h A|$. We start by recalling some basic terminology.

Definition 2.1. A standard graded algebra is a commutative algebra $R$ over a field $K$ endowed with a vector space decomposition $R=\oplus_{i \geq 0} R_{i}$ such that $R_{0}=K, R_{i} R_{j} \subseteq R_{i+j}$ for all $i, j \geq 0$, and which is generated as a K-algebra by finitely many elements in $R_{1}$.

It follows from the definition that each $R_{i}$ is a finite-dimensional vector space over $K$. Moreover, as $R$ is generated by $R_{1}$, we have $R_{i} R_{j}=R_{i+j}$ for all $i, j \geq 0$, whence $R_{i}=R_{1}^{i}$, the $i$-fold iterated productset of $R_{1}$.

Definition 2.2. Let $R=\oplus_{i \geq 0} R_{i}$ be a standard graded algebra. The Hilbert function of $R$ is the map $i \mapsto d_{i}$ associating to each $i \in \mathbb{N}$ the dimension

$$
d_{i}=\operatorname{dim}_{K} R_{i}
$$


of $R_{i}$ as a vector space over $K$.

Thus $d_{0}=1$, and $R$ is generated as a $K$-algebra by any $d_{1}$ linearly independent elements of $R_{1}$.

Let now $(G,+)$ be an abelian group. Consider the group algebra $K[G]$ of $G$. Its canonical $K$-basis is the set of symbols $\left\{t^{g} \mid g \in G\right\}$, and its product is induced by the formula

$$
t^{g_{1}} t^{g_{2}}=t^{g_{1}+g_{2}}
$$

for all $g_{1}, g_{2} \in G$. Consider now $S=K[G][Y]$, the one-variable polynomial algebra over $K[G]$. Then $S$ has for $K$-basis the set

$$
\mathcal{B}=\left\{t^{g} Y^{n} \mid g \in G, n \in \mathbb{N}\right\},
$$

and the product of any two basis elements is given by

$$
t^{g_{1}} Y^{n_{1}} \cdot t^{g_{2}} Y^{n_{2}}=t^{g_{1}+g_{2}} Y^{n_{1}+n_{2}}
$$

for all $g_{1}, g_{2} \in G$ and all $n_{1}, n_{2} \in \mathbb{N}$. The degree of a basis element is defined as

$$
\operatorname{deg}\left(t^{g} Y^{n}\right)=n
$$

for all $g \in G$ and all $n \in \mathbb{N}$. This endows $S$ with the structure of a graded algebra. Thus $S=\oplus_{h \geq 0} S_{h}$, where $S_{h}$ is the $K$-vector space with basis the set $\left\{t^{g} Y^{h} \mid g \in G\right\}$.

Definition 2.3. Let $A=\left\{a_{1}, \ldots, a_{n}\right\}$ be a nonempty finite subset of $G$. We define $R(A)$ to be the $K$-subalgebra of $S$ spanned by the set

$$
\left\{t^{a_{1}} Y, \ldots, t^{a_{n}} Y\right\} .
$$

Thus $R(A)$, being finitely generated over $K$ by elements of degree 1 , is a standard graded algebra. We then have $R=\oplus_{h \geq 0} R_{h}$, where $R_{h}$ is the $K$-vector space with basis the set $\left\{t^{b} Y^{h} \mid b \in h A\right\}$. It follows that

$$
\operatorname{dim} R_{h}=|h A|
$$

for all $h \geq 0$, as desired.

For future work on $R(A)$, it is algebraically important to determine the relations between its given generators $t^{a_{i}} Y$. This is done in Section 6 . 


\section{Macaulay's theorem}

We now turn to Macaulay's theorem [9] and a recent condensed version of it [3]. Macaulay's theorem gives a necessary and sufficient condition for a numerical function $\mathbb{N} \rightarrow \mathbb{N}$ to be the Hilbert function of some standard graded algebra. It rests on the so-called binomial representations of integers. Here is some background information.

Proposition 3.1. Let $a \geq i \geq 1$ be positive integers. There are unique integers $a_{i}>a_{i-1}>\cdots>a_{1} \geq 0$ such that

$$
a=\sum_{j=1}^{i}\left(\begin{array}{c}
a_{j} \\
j
\end{array}\right)
$$

Proof. See e.g. [1, 13].

This expression is called the $i$ th binomial representation of a. Producing it is computationally straightforward: take for $a_{i}$ the largest integer such that $\left(\begin{array}{c}a_{i} \\ i\end{array}\right) \leq a$, and complete $\left(\begin{array}{c}a_{i} \\ i\end{array}\right)$ by adding to it the $(i-1)$ th binomial representation of $a-\left(\begin{array}{c}a_{i} \\ i\end{array}\right)$. We omit trails of 0's, if any. For instance, for $a=10$ and $i=3$, we abbreviate $10=\left(\begin{array}{l}5 \\ 3\end{array}\right)+\left(\begin{array}{l}1 \\ 2\end{array}\right)+\left(\begin{array}{l}0 \\ 1\end{array}\right)$ as simply $10=\left(\begin{array}{l}5 \\ 3\end{array}\right)$.

Notation 3.2. Let $a \geq i \geq 1$ be positive integers. Let $a=\sum_{j=1}^{i}\left(\begin{array}{c}a_{j} \\ j\end{array}\right)$ be its ith binomial representation. We denote $a^{\langle i\rangle}=\sum_{j=1}^{i}\left(\begin{array}{c}a_{j}+1 \\ j+1\end{array}\right)$ and $0^{\langle i\rangle}=0$.

Note that the defining formula of $a^{\langle i\rangle}$ yields the $(i+1)$ th binomial representation of the integer it sums to.

Here is one half of Macaulay's classical result, constraining the possible Hilbert functions of standard graded algebras [9].

Theorem 3.3. Let $R=\oplus_{i \geq 0} R_{i}$ be a standard graded algebra over a field $K$, with Hilbert function $d_{i}=\operatorname{dim}_{K} R_{i}$. Then for all $i \geq 1$, we have

$$
d_{i+1} \leq d_{i}^{\langle i\rangle}
$$

Remarkably, the converse also holds in Macaulay's theorem, but we shall not need it here. That is, satisfying (4) for all $i \geq 1$ characterizes the Hilbert functions of standard graded algebras. See e.g. [1, 10, 13. 
Example 3.4. Consider the sequence

$$
\left(m_{0}, m_{1}, m_{2}, m_{3}, m_{4}, m_{5}, m_{6}\right)=(1,5,15,33,61,100,152) .
$$

Then $m_{i+1} \leq m_{i}^{\langle i\rangle}$ for all $i=1, \ldots, 5$ as readily checked. Hence there exists a standard graded algebra $R=\oplus_{j \geq 0} R_{j}$ whose values of $\operatorname{dim} R_{i}$ for $i=0, \ldots, 6$ are exactly modeled by the sequence $\left(m_{0}, \ldots, m_{6}\right)$. For instance, one may take $R=S / J$, where $S=K\left[X_{1}, \ldots, X_{5}\right]$ and $J=\left(X_{5}^{3}, X_{4} X_{5}^{2}, X_{3}^{3} X_{5}^{2}\right)$.

\subsection{A condensed version}

For our new derivation of the Plünnecke-based estimate (1), we shall need the following condensed version of Macaulay's theorem established in [3]. For $m \in \mathbb{N}$ and $x \in \mathbb{R}$, denote as usual

$$
\left(\begin{array}{c}
x \\
m
\end{array}\right)=\frac{x(x-1) \cdots(x-m+1)}{m !}=\prod_{i=0}^{m-1} \frac{x-i}{m-i} .
$$

In particular, $\left(\begin{array}{l}x \\ 0\end{array}\right)=1$. We shall constantly need the following observations.

Lemma 3.5. Let $i \geq 1$ be an integer. Then the map $y \mapsto\left(\begin{array}{c}y \\ i\end{array}\right)$ is an increasing continuous bijection (in fact, a homeomorphism) from $[i-1, \infty)$ to $[0, \infty)$. In particular, for any real numbers $y_{1}, y_{2} \geq i-1$, we have

$$
y_{1} \leq y_{2} \Longleftrightarrow\left(\begin{array}{c}
y_{1} \\
i
\end{array}\right) \leq\left(\begin{array}{c}
y_{2} \\
i
\end{array}\right)
$$

Proof. A direct consequence of Rolle's theorem. See e.g. [3, Lemma 5.6].

Lemma 3.6. Let $h, d \geq 1$ be positive integers. Then there exists a unique real number $x \geq h$ such that $d=\left(\begin{array}{l}x \\ h\end{array}\right)$.

Proof. By the above lemma, there is a unique real number $x \geq h-1$ such that $d=\left(\begin{array}{l}x \\ h\end{array}\right)$. Since $d \geq 1$, we have $\left(\begin{array}{l}x \\ h\end{array}\right) \geq\left(\begin{array}{l}h \\ h\end{array}\right)$. Hence $x \geq h$ by (5).

Here is the condensed version of Macaulay's theorem that we shall use in the next section. 
Theorem 3.7. Let $R=\oplus_{i \geq 0} R_{i}$ be a standard graded algebra over the field $\mathbb{K}$, with Hilbert function $d_{i}=\operatorname{dim}_{\mathbb{K}} R_{i}$ for $i \geq 0$. Let $h \geq 1$ be an integer. Let $x \geq h-1$ be the unique real number such that $d_{h}=\left(\begin{array}{l}x \\ h\end{array}\right)$. Then

$$
d_{h-1} \geq\left(\begin{array}{l}
x-1 \\
h-1
\end{array}\right) \text { and } d_{h+1} \leq\left(\begin{array}{l}
x+1 \\
h+1
\end{array}\right)
$$

Proof. See [3].

\section{Main results}

Let $A$ be a finite subset of an abelian group with $|A| \geq 2$. If $|h A|$ is known for some $h \geq 2$, what bounds can one derive on $|i A|$ for $i \neq h$ ?

We first recall the classical known answer, a direct consequence of Plünnecke's inequality. See e.g. [11, Theorem 7.5, p. 217] or [16, Theorem 1.2.3 with $m=1$, p. 96].

Theorem 4.1 (Plünnecke). Let $A$ be a nonempty finite subset of an abelian group. Let $h \geq 2$ be an integer. Then $|i A| \geq|h A|^{i / h}$ for all $1 \leq i \leq h$.

Remark 4.2. Theorem 4.1 is equivalent to its main case $i=h-1$, namely:

$$
|(h-1) A| \geq|h A|^{(h-1) / h} .
$$

Indeed, the general case is implied by (6), as shown by induction on $h$ :

$$
|i A| \stackrel{\sqrt[6]{ } \geq}{\geq}\left(|(i+1) A|^{i /(i+1)} \stackrel{\text { ind.hyp. }}{\geq}\left(|h A|^{(i+1) / h}\right)^{i /(i+1)}=|h A|^{i / h} .\right.
$$

Consequently, in the sequel, we mainly focus on comparing $|h A|$ with $|(h-1) A|$ and/or $|(h+1) A|$. In this spirit, a particular case of Plünnecke's Theorem 4.1 is the estimate

$$
|(h+1) A| \leq|h A|^{(h+1) / h}
$$

for all $h \geq 1$.

In comparison, here is our first main result, obtained by applying Macaulay's Theorem 3.3 to the standard graded algebra $R(A)$ defined in Section 2 . 
Theorem 4.3. Let $A$ be a nonempty finite subset of an abelian group $G$. Let $h \geq 1$ be an integer. Then

$$
|(h+1) A| \leq|h A|^{\langle h\rangle} .
$$

The strength of Theorem 4.3 is illustrated in Section 4.1, with a concrete example showing that (8) may be much sharper than (7). In fact, the improvement of the former over the latter is systematic, as shown by Theorem 4.9, Corollary 4.10 and Remark 4.11. See also a comment in Section 7 .

Proof. Let $R=R(A)$ be the standard graded algebra associated to $A$ as defined in Section 2. We have $R=\oplus_{h \geq 0} R_{h}$, where $R_{h}$ denotes the homogeneous subspace of $R$ of degree $h$. By (3) we have

$$
\operatorname{dim} R_{h}=|h A|
$$

for all $h \geq 0$. Hence, for $h \geq 1$, a direct application of Theorem 3.3 yields the claimed upper bound (8).

Let us now apply Theorem 3.7, the condensed version of Macaulay's theorem. We obtain the following more flexible bounds from which we shall derive and improve (6).

Theorem 4.4. Let $A$ be a nonempty finite subset of an abelian group $G$. Let $h \geq 2$ be an integer and $x \geq h$ the unique real number such that $|h A|=\left(\begin{array}{l}x \\ h\end{array}\right)$. Then

$$
|(h-1) A| \geq\left(\begin{array}{l}
x-1 \\
h-1
\end{array}\right) \text { and }|(h+1) A| \leq\left(\begin{array}{l}
x+1 \\
h+1
\end{array}\right) .
$$

Proof. As above, let $R=R(A)$ be the standard graded algebra associated to $A$ with its decomposition $R=\oplus_{h \geq 0} R_{h}$ into the direct sum of its homogeneous subspaces of given degree, where $\operatorname{dim} R_{h}=|h A|$ for all $h \geq 0$. The claimed bounds follow from Theorem 3.7 applied to $R(A)$.

Remark 4.5. While more handy, the upper bound in Theorem 4.4 is slightly weaker than in Theorem 4.3. Indeed, still with $x \geq h$ such that $|h A|=\left(\begin{array}{l}x \\ h\end{array}\right)$, we have

$$
|h A|^{\langle h\rangle} \leq\left(\begin{array}{l}
x+1 \\
h+1
\end{array}\right) .
$$

This follows from [3, Theorem 5.9]. 
Given $|h A|$, the lower bound on $|(h-1) A|$ provided by Theorem 4.4 may be up to 2.71 times better, in suitable circumstances, than the one provided in (6) by Plünnecke's inequality. This will be shown in Sections 4.2 and 5 . We start with a concrete example demonstrating the strength of Theorems 4.3 and 4.4 .

\subsection{An example: the case $|5 A|=100$}

Let $A$ be a subset of an abelian group such that $|5 A|=100$. The Plünneckebased bounds given by (6), namely $|4 A| \geq 100^{4 / 5}$ and $|6 A| \leq 100^{6 / 5}$, yield

$$
|4 A| \geq 40, \quad|6 A| \leq 251
$$

In comparison, based on the condensed version of Macaulay's theorem, Theorem 4.4 yields the much sharper bounds

$$
|4 A| \geq 58, \quad|6 A| \leq 161
$$

Indeed, let $x \geq 5$ be the unique real number such that $\left(\begin{array}{l}x \\ 5\end{array}\right)=100$. Then $8.69<x<8.7$, as follows from $\left(\begin{array}{c}8.69 \\ 5\end{array}\right) \approx 99.42$ and $\left(\begin{array}{c}8.7 \\ 5\end{array}\right) \approx 100.2$. Hence

$$
\begin{aligned}
& |4 A| \geq\left(\begin{array}{c}
x-1 \\
4
\end{array}\right)>\left(\begin{array}{c}
7.69 \\
4
\end{array}\right) \approx 57.2 \\
& |6 A| \leq\left(\begin{array}{c}
x+1 \\
6
\end{array}\right)<\left(\begin{array}{c}
9.7 \\
6
\end{array}\right) \approx 161.99
\end{aligned}
$$

This proves (9). Theorem 4.3, based on the full version of Macaulay's theorem, yields even better bounds.

Proposition 4.6. Let $A$ be a subset of an abelian group such that $|5 A|=100$. Then

$$
|4 A| \geq 61, \quad|6 A| \leq 152 .
$$

Proof. The 5th binomial representation of 100 is given by

$$
100=\left(\begin{array}{l}
8 \\
5
\end{array}\right)+\left(\begin{array}{l}
7 \\
4
\end{array}\right)+\left(\begin{array}{l}
4 \\
3
\end{array}\right)+\left(\begin{array}{l}
3 \\
2
\end{array}\right)+\left(\begin{array}{l}
2 \\
1
\end{array}\right) .
$$


The inequality $|(h+1) A| \leq|h A|^{\langle h\rangle}$ of Theorem 4.3 then yields 10$)$. More precisely, we have

$$
\begin{aligned}
& |4 A| \geq\left(\begin{array}{l}
7 \\
4
\end{array}\right)+\left(\begin{array}{l}
6 \\
3
\end{array}\right)+\left(\begin{array}{l}
4 \\
2
\end{array}\right)=61 \\
& |6 A| \leq\left(\begin{array}{l}
9 \\
6
\end{array}\right)+\left(\begin{array}{l}
8 \\
5
\end{array}\right)+\left(\begin{array}{l}
5 \\
4
\end{array}\right)+\left(\begin{array}{l}
4 \\
3
\end{array}\right)+\left(\begin{array}{l}
3 \\
2
\end{array}\right)=152
\end{aligned}
$$

Thus inequality (12) directly follows from Theorem 4.3. As for (11), if we had $|4 A| \leq 60=\left(\begin{array}{l}7 \\ 4\end{array}\right)+\left(\begin{array}{l}6 \\ 3\end{array}\right)+\left(\begin{array}{l}3 \\ 2\end{array}\right)+\left(\begin{array}{l}2 \\ 1\end{array}\right)$, then that same theorem would imply $|5 A| \leq\left(\begin{array}{l}8 \\ 5\end{array}\right)+\left(\begin{array}{l}7 \\ 4\end{array}\right)+\left(\begin{array}{l}4 \\ 3\end{array}\right)+\left(\begin{array}{l}3 \\ 2\end{array}\right)=100-2$, contrary to the hypothesis.

Is Proposition 4.6 best possible for sets satisfying $|5 A|=100$ ? Strong evidence shows that it is not far from it. For instance, let $A=\{0,1,5,8,49\} \subset$ $\mathbb{Z}$. Then $|5 A|=100$ as required, and

$$
|4 A|=63, \quad|6 A|=145 .
$$

We conjecture that these bounds are optimal for sets of integers.

Conjecture 4.7. Let $A \subset \mathbb{Z}$ be any subset satisfying $|5 A|=100$. Then $|4 A| \geq 63$ and $|6 A| \leq 145$.

As seen here, the improvement provided by Theorem 4.4 is already quite good. How good is it in general? We investigate this question in the sequel.

\subsection{Macaulay vs Plünnecke}

As our next main result, we show that Plünnecke's Theorem 4.1 also follows from our Macaulay-based Theorem 4.4, and we significantly strengthen it by a multiplicative factor which may exceed 2.71 in suitable circumstances.

Notation 4.8. For a positive integer $h$ and a real number $x \geq h$, we set

$$
\theta(x, h)=\frac{h}{x}\left(\begin{array}{l}
x \\
h
\end{array}\right)^{1 / h} .
$$


Theorem 4.9. Let $A$ be a nonempty finite subset of an abelian group $G$. Let $h \geq 2$ be an integer. Then

$$
|(h-1) A| \geq \theta(x, h)|h A|^{(h-1) / h},
$$

where $x \in \mathbb{R}$ satisfies $x \geq h$ and $|h A|=\left(\begin{array}{l}x \\ h\end{array}\right)$.

Proof. Theorem 4.4 yields

$$
|(h-1) A| \geq\left(\begin{array}{l}
x-1 \\
h-1
\end{array}\right) .
$$

Now

$$
\left(\begin{array}{l}
x-1 \\
h-1
\end{array}\right)=\frac{h}{x}\left(\begin{array}{l}
x \\
h
\end{array}\right)
$$

since

$$
\left(\begin{array}{l}
x \\
h
\end{array}\right)=\prod_{i=0}^{h-1} \frac{x-i}{h-i}=\frac{x}{h} \prod_{i=1}^{h-1} \frac{x-i}{h-i}=\frac{x}{h}\left(\begin{array}{l}
x-1 \\
h-1
\end{array}\right) .
$$

Hence

$$
\begin{aligned}
|(h-1) A|^{h} & \geq\left(\begin{array}{l}
x-1 \\
h-1
\end{array}\right)^{h} \\
& =\left(\frac{h}{x}\right)^{h}\left(\begin{array}{l}
x \\
h
\end{array}\right)^{h} \\
& =\left(\frac{h}{x}\right)^{h}\left(\begin{array}{l}
x \\
h
\end{array}\right)\left(\begin{array}{l}
x \\
h
\end{array}\right)^{h-1} \\
& =\left(\frac{h}{x}\right)^{h}\left(\begin{array}{l}
x \\
h
\end{array}\right)|h A|^{h-1} .
\end{aligned}
$$

Therefore $|(h-1) A|^{h} \geq \theta(x, h)^{h}|h A|^{h-1}$, as desired.

Corollary 4.10. Theorem 4.4 implies Plünnecke's Theorem 4.1.

Proof. By Theorem 4.9, we only need to show $\theta(x, h) \geq 1$, or equivalently, $\theta(x, h)^{h} \geq 1$. Now

$$
\theta(x, h)^{h}=\left(\frac{h}{x}\right)^{h}\left(\begin{array}{l}
x \\
h
\end{array}\right)=\prod_{i=0}^{h-1} \frac{h(x-i)}{x(h-i)},
$$

and $h(x-i) \geq x(h-i)$ for all $0 \leq i \leq h-1$ since $h \leq x$. 
Remark 4.11. In fact, we have $\theta(x, h)>1$ whenever $h \geq 2$ and $|h A| \geq 2$. Inded, since $|h A|=\left(\begin{array}{l}x \\ h\end{array}\right)$ with $x \geq h$, it follows that $x>h$, whence $h(x-1)>$ $x(h-1)$, implying in turn $\theta(x, h)^{h}>1$ by 15 .

We close this section with an equivalent formulation of Theorem 4.9. It provides a nice inequality between $|(h-1) A|$ and $|h A|$, yet less suited to comparison purposes with Plünnecke's inequality.

Theorem 4.12. Let $A$ be a nonempty finite subset of an abelian group $G$. Let $h \geq 2$ be an integer. Then

$$
|(h-1) A| \geq \frac{h}{x}|h A|,
$$

where $x \in \mathbb{R}$ satisfies $x \geq h$ and $|h A|=\left(\begin{array}{l}x \\ h\end{array}\right)$.

Proof. Directly follows from Theorem 4.9 and the formulas

$$
\begin{aligned}
\theta(x, h) & =\frac{h}{x}\left(\begin{array}{l}
x \\
h
\end{array}\right)^{1 / h} \\
& =\frac{h}{x}|h A|^{(h-1) / h} .
\end{aligned}
$$

Alternatively, directly follows from Theorem 4.4 and formula (14).

Corollary 4.13. Let $A$ be a nonempty finite subset of an abelian group $G$. For all $i \geq 1$, let $x_{i} \in \mathbb{R}$ satisfy $x_{i} \geq i$ and $\left(\begin{array}{c}x_{i} \\ i\end{array}\right)=|i A|$. Let $h \geq 2$ be an integer. For all $1 \leq i \leq h-1$, we have

$$
|h A| \leq\left(\prod_{j=i+1}^{h} x_{j} / j\right)|i A| .
$$

Proof. Straightforward consequence of the above theorem.

\section{Behavior of $\theta(x, h)$}

We now study the numerical behavior of the function $\theta(x, h)$. Denote $e \approx$ 2.718 , the basis of the natural logarithm. We show that $1<\theta(x, h)<e$ whenever $x>h \geq 2$, and that $\theta(x, h)$ asymptotically tends to $e$ in suitable circumstances. This section is slightly more informal in nature. Numerical computations and graphics were done with Mathematica 10 [20]. 
Proposition 5.1. For all $h \in \mathbb{N}, x \in \mathbb{R}$ such that $x>h \geq 2$, we have

$$
1<\theta(x, h)<e .
$$

Proof. The lower bound follows from (15) and Remark 4.11. As for the upper bound, we have

$$
\left(\begin{array}{l}
x \\
h
\end{array}\right) \leq \frac{x^{h}}{h !}=\frac{x^{h}}{h^{h}} \frac{h^{h}}{h !}<\frac{x^{h}}{h^{h}} e^{h}
$$

since $\frac{h^{h}}{h !}<\sum_{k \in \mathbb{N}} \frac{h^{k}}{k !}=e^{h}$. It follows that

$$
\theta(x, h)=\frac{h}{x}\left(\begin{array}{l}
x \\
h
\end{array}\right)^{1 / h}<\frac{h}{x} \frac{x}{h} e=e .
$$

We shall also need to invoke the monotonicity of $\theta(x, h)$ in $x$.

Proposition 5.2. For a fixed integer $h \geq 2$, the map $x \mapsto \theta(x, h)$ from $[h, \infty)$ to $[1, \infty)$ is strictly increasing.

Proof. It is equivalent to show that the map $x \mapsto \theta(x, h)^{h}$ is strictly increasing. This easily follows from the positivity of its derivative. Details are left to the reader.

\section{$5.1 \quad$ Asymptotics}

We provide here, somewhat informally, a good approximation of $\theta(x, h)$ together with its asymptotic behavior as $x$ grows. Recall Stirling's approximation of $n$ ! for large $n$ :

$$
n ! \sim \sqrt{2 \pi n}\left(\frac{n}{e}\right)^{n} .
$$

On the other hand, the bounds below are valid for all $n \geq 1$ :

$$
\sqrt{2 \pi} n^{n+1 / 2} e^{-n} \leq n ! \leq e n^{n+1 / 2} e^{-n} .
$$

This yields the following well known approximation of $\left(\begin{array}{l}n \\ k\end{array}\right)$ for $n$ much larger than $k$, see e.g. [19]:

$$
\left(\begin{array}{l}
n \\
k
\end{array}\right) \sim \frac{(n / k-1 / 2)^{k} e^{k}}{\sqrt{2 \pi k}}
$$

As a consequence, here is the asymptotic behavior of $\theta(x, h)$ when $x$ grows. 
Proposition 5.3. Let $h \geq 2$ be an integer. Then

$$
\theta(x, h) \sim \frac{(1-h /(2 x)) e}{(2 \pi h)^{1 /(2 h)}}=\frac{(2 x-h) e}{2 x(2 \pi h)^{1 /(2 h)}} .
$$

In particular,

$$
\lim _{x \rightarrow \infty} \theta(x, h)=(2 \pi h)^{-1 /(2 h)} e .
$$

Proof. Directly follows from the above approximation of the binomial coefficients.

\subsection{When $\theta(x, h) \geq 1.5$}

Our multiplicative improvement factor $\theta(x, h)$ over the Plünnecke-based estimate

$$
|(h-1) A| \geq|h A|^{(h-1) / h}
$$

exceeds 1.5 quite early in terms of $x$ or $h$. Indeed, one observes that the smallest integer $x$ for which $\theta(x, h) \geq 1.5$ for some integer $h$ is $x=10$, specifically at $h=4$ and 5 . Even starting at $h=3$, we have

$$
\theta(x, 3) \geq 1.509
$$

for all $x \geq 12$. As an example of application, these observations, together with Theorem 4.9, yield the following improvements of 16 for $h=3,4,5$.

Corollary 5.4. Let $A$ be a finite subset of an abelian group $G$. Then

$$
\begin{aligned}
|3 A| \geq 220 & \Longrightarrow|2 A| \geq \frac{3}{2}|3 A|^{2 / 3}, \\
|4 A| \geq 210 & \Longrightarrow|3 A| \geq \frac{3}{2}|4 A|^{3 / 4}, \\
|5 A| \geq 252 & \Longrightarrow|4 A| \geq \frac{3}{2}|5 A|^{4 / 5} .
\end{aligned}
$$

Proof. For $h=3$, let $x$ be the unique real number greater than 3 such that $|3 A|=\left(\begin{array}{l}x \\ 3\end{array}\right)$. Since $|3 A| \geq 220=\left(\begin{array}{c}12 \\ 3\end{array}\right)$, we have $x \geq 12$, whence $\theta(x, 3) \geq 1.5$ by (17). The conclusion follows from Theorem 4.9. For $h=4$ and 5, we have $\left(\begin{array}{c}10 \\ 4\end{array}\right)=210$ and $\left(\begin{array}{c}10 \\ 5\end{array}\right)=252$. The rest of the proof is similar, using the above-mentioned estimates $\theta(x, 4), \theta(x, 5) \geq 1.5$ for all $x \geq 10$. 


\subsection{When $\theta(x, h) \geq 2$}

We now examine circumstances guaranteeing $\theta(x, h) \geq 2$, a case of interest where our bound in Theorem 4.9 is at least twice better than (16). As it turns out, for $x$ large, one has $\theta(x, h) \geq 2$ for almost all integers $h$ between 6 and $\lfloor x / 2\rfloor$. We also describe cases where $\theta(x, h)$ gets very close to its upper bound $e$.

So, under what minimal circumstances, in terms of $h$ or of $x$, do we have $\theta(x, h) \geq 2$ ? First note that if $y \geq h-1$ then $\theta(y, h)<\lim _{x \rightarrow \infty} \theta(x, h)$, as follows from Proposition 5.2. Moreover, $\lim _{x \rightarrow \infty} \theta\left(x, h_{1}\right) \leq \lim _{x \rightarrow \infty} \theta\left(x, h_{2}\right)$ whenever $h_{1} \leq h_{2}$, as follows from Proposition 5.3 .

That being said, consider the case $h=5$. Since $\lim _{x \rightarrow \infty} \theta(x, 5)<1.926$ by Proposition 5.3, the values $1 \leq h \leq 5$ are excluded for the occurrence of $\theta(x, h) \geq 2$. However, already $h=6$ qualifies, as $\lim _{x \rightarrow \infty} \theta(x, 6)>2.007$. More precisely, we have

$$
\theta(x, 6) \geq 2
$$

for all $x \geq 1210$, the least integer with that property.

If now $h$ is allowed to grow, then $\theta(x, h) \geq 2$ may occur for much smaller values of $x$. Indeed, the smallest $x \in \mathbb{N}$ for which $\theta(x, h) \geq 2$ for some $h$ is $x=48$, namely at $h=11$ and 12 . More precisely, we have

$$
\begin{aligned}
& \theta(48,11)>2.001, \quad \theta(48,12)>2.002 \\
& \theta(48,10)<1.997, \quad \theta(48,13)<1.999 .
\end{aligned}
$$

See Figure 1

In fact, when $x$ goes to infinity, then $\theta(x, h) \geq 2$ holds for almost all positive integers $h \leq x / 2$. Indeed, as observed in (18), we have $\theta(x, 6) \geq 2$ for all $x \geq x_{0}=1210$. Now, numerical computations at $x_{0}$ yield

$$
\theta\left(x_{0}, h\right) \geq 2 \quad \forall h \in\left[6, x_{0} / 2-10\right] \cap \mathbb{N} .
$$

Together with Theorem 4.9, this yields the following factor 2 improvement over the Plünnecke-based estimate (16).

Corollary 5.5. Let $h$ be an integer such that $6 \leq h \leq 595$. Let $A$ be a subset of an abelian group $G$ such that $|h A| \geq\left(\begin{array}{c}2 h+20 \\ h\end{array}\right)$. Then

$$
|(h-1) A| \geq 2|h A|^{(h-1) / h} \text {. }
$$




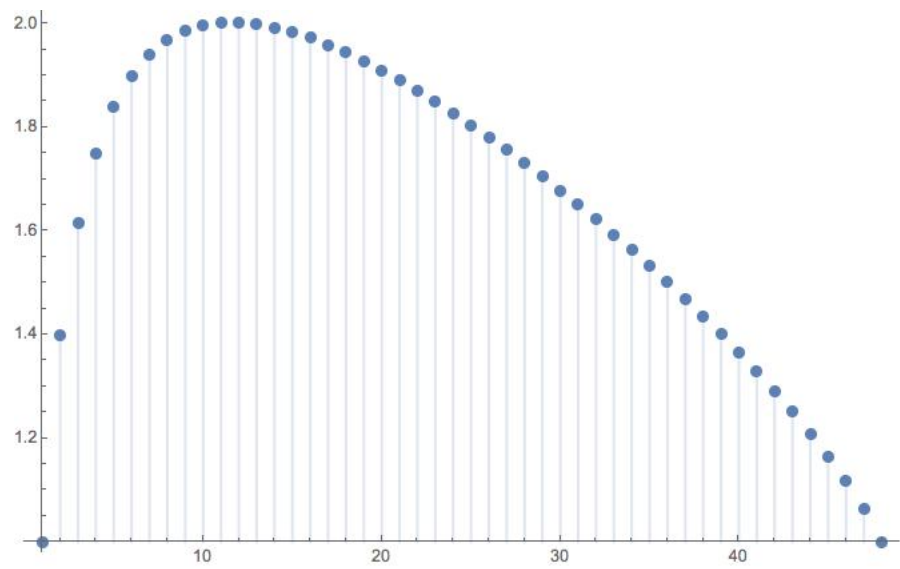

Figure 1: Values of $\theta(48, h)$ for $h=1, \ldots, 48$

Proof. Let $x \geq h$ satisfy $|h A|=\left(\begin{array}{l}x \\ h\end{array}\right)$. Since $|h A| \geq\left(\begin{array}{c}2 h+20 \\ h\end{array}\right)$, it follows that $x \geq 2 h+20 \geq 1210=x_{0}$. Hence $h \in\left[6, x_{0} / 2-10\right] \cap \mathbb{N}$, whence $\theta(x, h) \geq 2$ by (19) and Proposition 5.2. The conclusion follows from Theorem 4.9.

As yet another instance, for $x_{1}=10^{6}$ now, one has an almost identical statement as in 19 for $x_{0}=1210$, namely

$$
\theta\left(x_{1}, h\right) \geq 2 \quad \forall h \in\left[6, x_{1} / 2-19\right] \cap \mathbb{N} .
$$

Statements (19) and (20) are no accident, as hinted by the following result.

Proposition 5.6. One has $\lim _{x \rightarrow \infty} \theta(x,\lfloor x / 2\rfloor)=2$.

Proof. Using Stirling's approximation formula of $n$ !, one readily sees that

$$
\theta(n,\lfloor n / 2\rfloor) \approx 2\left(\frac{2}{\pi n}\right)^{1 / n}
$$

which proves the claim since $\lim _{n \rightarrow \infty}(c n)^{-1 / n}=1$ for any constant $c>0$.

\subsection{The highest point}

For fixed $x$, the general shape of $\theta(x, h)$ when $h$ runs from 1 to $\lfloor x\rfloor$ is well illustrated by Figure 1 for $x=48$. Figure 2 displays the case $x=1000$. 


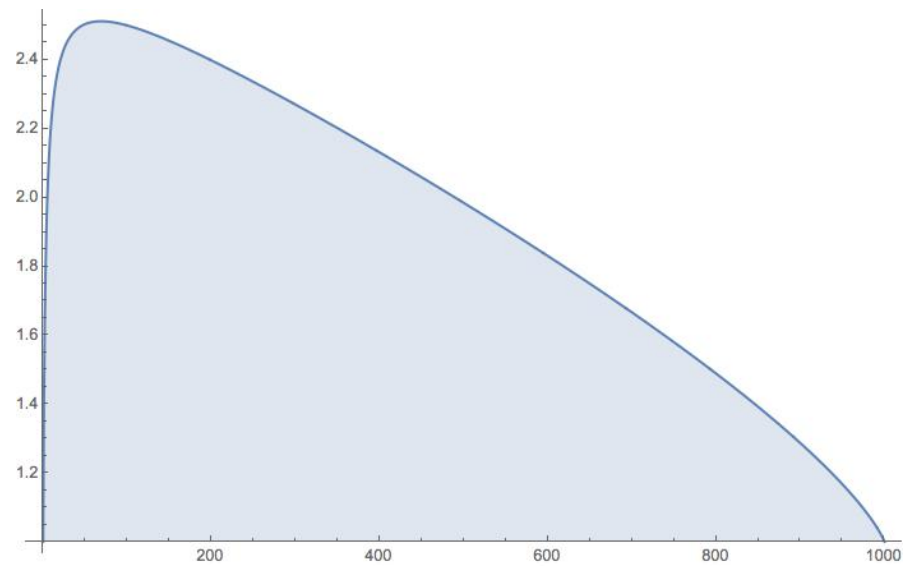

Figure 2: Values of $\theta(1000, h)$ for $h=1, \ldots, 1000$

It would be desirable to determine the highest point of that curve, and in particular the integer $1 \leq h \leq x$ maximizing $\theta(x, h)$. We do not have yet a precise answer. Nevertheless, by computing derivatives of the approximation of $\theta(x, h)$ provided by Proposition 5.3, one sees that for fixed $x$,

$$
\frac{\partial}{\partial h}\left(\frac{2 x-h}{(2 \pi h)^{1 /(2 h)}}\right)>0 \Longleftrightarrow 2 h^{2}<(2 x-h)(\ln (2 \pi h)-1) .
$$

Thus, for $x$ fixed, the sought-for integer $h$ maximizing $\theta(x, h)$ occurs when

$$
2 h^{2} \approx(2 x-h)(\ln (2 \pi h)-1) .
$$

For instance, for $x_{0}=100$, the maximum of $\theta\left(x_{0}, h\right)$ is reached at $h=18$, for which $\theta(100,18) \approx 2.177$. Hence

$$
\theta(x, 18) \geq 2.177
$$

for all $x \geq 100$, as follows from Proposition 5.2 .

\subsection{For $h$ fixed}

In the opposite direction, for $h$ fixed, it is easy to locate the real number $x_{1} \geq h$ maximizing $\theta(x, h)$. Indeed, using (21), we find

$$
x_{1} \approx \frac{1}{2}\left(\frac{2 h^{2}}{\ln (2 \pi h)-1}+h\right) .
$$


This suggests that

$$
\lim _{x \rightarrow \infty} \theta\left(x,\left\lfloor x^{1 / 2}\right\rfloor\right)=e,
$$

as is fully confirmed by numerical experiments. As a concrete illustration, here are instances where $\theta(x, h)$ gets very close to $e$ :

- For all $x \geq 200000$ and all $1200 \leq h \leq 1300$, one has $\theta(x, h) \geq 2.70$.

- Similarly, for all $x \geq 1100000$ and all $2600 \leq h \leq 3700$, one has $\theta(x, h) \geq 2.71$.

\section{$6 \quad$ A presentation of $R(A)$}

Reusing the notation of Section 2 , let $A=\left\{a_{1}, \ldots, a_{n}\right\}$ be a nonempty finite subset of an abelian group $(G,+)$. For future use, it is algebraically necessary to determine the relations between the given generators $t^{a_{i}} Y$ of the associated algebra $R(A)$. Our aim here is thus to identify $R(A)$ as the quotient of the polynomial algebra $K\left[X_{1}, \ldots, X_{n}\right]$ by a suitable homogeneous ideal $I$.

Notation 6.1. For $\alpha=\left(\alpha_{1}, \ldots, \alpha_{n}\right) \in \mathbb{N}^{n}$, let $X^{\alpha}=X_{1}^{\alpha_{1}} \cdots X_{n}^{\alpha_{n}}$ denote the corresponding monomial in $K\left[X_{1}, \ldots, X_{n}\right]$. We denote the set of these monomials by $M=\left\{X^{\alpha} \mid \alpha \in \mathbb{N}^{n}\right\}$.

Let $\varphi: K\left[X_{1}, \ldots, X_{n}\right] \rightarrow R(A)$ be the surjective morphism induced by $\varphi\left(X_{i}\right)=t^{a_{i}} Y$ for all $i$. On the set $M$, we define the equivalence relation

$$
u \sim v \Longleftrightarrow \varphi(u)=\varphi(v)
$$

for all $u, v \in M$. Equivalently, let us write $u=X^{\alpha}, v=X^{\beta}$ with $\alpha=$ $\left(\alpha_{1}, \ldots, \alpha_{n}\right), \beta=\left(\beta_{1}, \ldots, \beta_{n}\right) \in \mathbb{N}^{n}$. Then

$$
X^{\alpha} \sim X^{\beta} \Longleftrightarrow \begin{cases}\sum_{i} \alpha_{i} & =\sum_{i} \beta_{i}, \\ \sum_{i} \alpha_{i} a_{i} & =\sum_{i} \beta_{i} a_{i} .\end{cases}
$$

In particular, equivalent monomials have the same degree, where as usual $\operatorname{deg}\left(X^{\alpha}\right)=\sum_{i} \alpha_{i}$.

We shall need the notion of simple polynomial relative to $\sim$.

Definition 6.2. Let $f \in K\left[X_{1}, \ldots, X_{n}\right]$. We say that $f$ is simple if $f \neq 0$ and all monomials occurring in $f$ are equivalent under $\sim$. 
Observe that a simple polynomial is homogeneous. Indeed, equivalent monomials under $\sim$ have the same degree as observed above. Moreover, every nonzero polynomial $g \in K\left[X_{1}, \ldots, X_{n}\right]$ may be decomposed, in a unique way up to order, as the sum $g=f_{1}+\cdots+f_{r}$ of maximal simple polynomials $f_{i}$, in the sense that for all $i \neq j$, the monomials occurring in $f_{i}$ are nonequivalent under $\sim$ to those of $f_{j}$. The $f_{i}$ are obtained by simply regrouping the monomials of $f$ into maximal equivalence classes. We shall refer to the $f_{i}$ as the simple components of $f$. See e.g. [2, p. 232] and [5, p. 346], where similar notions were used.

Lemma 6.3. Let $g \in \operatorname{ker}(\varphi) \backslash\{0\}$. Then every simple component of $g$ belongs to $\operatorname{ker}(\varphi)$.

Proof. Let $f$ be a simple component of $g$. We must show $\varphi(f)=0$. Since $f$ is simple, it is homogeneous of some degree $h$. Write $f=\sum_{i} \lambda_{i} u_{i}$, where $\lambda_{i} \in K \backslash\{0\}$ for all $i$ and where the $u_{i}$ are pairwise distinct monomials. Since the $u_{i}$ are pairwise equivalent under $\sim$, we have $\varphi\left(u_{i}\right)=t^{b} Y^{h}$ for some $b \in h A$ independent of $i$. Hence

$$
\varphi(f)=\left(\sum_{i} \lambda_{i}\right) t^{b} Y^{h}
$$

Now, for any monomial $v$ occurring in $g$ but not in $f$, we have $\varphi(v) \neq t^{b} Y^{h}$ as $v$ is non-equivalent to the $u_{i}$. Since $\varphi(g)=0$, it follows that $\sum_{i} \lambda_{i}=0$. Hence $\varphi(f)=0$, as desired.

Proposition 6.4. Let $I \subset K\left[X_{1}, \ldots, X_{n}\right]$ be the ideal generated by the set $\{u-v \mid u, v \in M, u \sim v\}$. Then $\operatorname{ker}(\varphi)=I$.

Proof. We have $I \subset \operatorname{ker}(\varphi)$ by construction. Conversely, let $0 \neq f \in \operatorname{ker}(\varphi)$. By Lemma 6.3, we may further assume that $f$ is simple. Write $f=\sum_{i=1}^{r} \lambda_{i} u_{i}$, where $\lambda_{i} \in K \backslash\{0\}$ for all $i$ and where the $u_{i}$ are pairwise distinct monomials. Since $\varphi(f)=0$ and $\varphi\left(u_{i}\right)=\varphi\left(u_{j}\right)$ for all $i \neq j$, it follows that $\sum_{i=1}^{r} \lambda_{i}=0$. Therefore $\lambda_{r}=-\sum_{i=1}^{r-1} \lambda_{i}$, and so

$$
f=\sum_{i=1}^{r-1} \lambda_{i}\left(u_{i}-u_{r}\right) .
$$

Since $u_{i} \sim u_{r}$ for all $i$, it follows that $u_{i}-u_{r} \in I$. Hence $f \in I$, as desired.

Corollary 6.5. We have $R(A) \simeq K\left[X_{1}, \ldots, X_{n}\right] / I$.

Proof. By Noether's isomorphism theorem. 


\section{Concluding comments}

We end this paper with a few related questions and remarks.

A first natural question is, how far from optimal are our new bounds? More precisely, let $(G,+)$ be an abelian group, and let $h, i, m$ be positive integers such that $m \leq|G|$. Among all subsets $A \subseteq G$ such that $|h A|=m$, what is

- (inverse problem) the best possible lower bound on $|i A|$ for $i \leq h$ ?

- (direct problem) the best possible upper bound on $|i A|$ for $i \geq h$ ?

Accordingly, let us denote

$$
\omega_{G}(h, i, m)= \begin{cases}\min _{A \subseteq G}|i A| & \text { if } i \leq h, \\ \max _{A \subseteq G}|i A| & \text { if } i \geq h\end{cases}
$$

where in both cases, the extremum is taken over all subsets $A$ of $G$ satisfying $|h A|=m$.

Focusing here on the direct problem with $i=h+1$, how large can $\omega_{G}(h, h+1, m)$ be? The upper bounds given successively by Plünnecke's inequality (7), Theorem 4.4 based on the condensed version of Macaulay's theorem, and Theorem 4.3 based on Macaulay's theorem proper, are

$$
\begin{aligned}
\omega_{G}(h, h+1, m) & \leq m^{(h+1) / h} \\
\omega_{G}(h, h+1, m) & \leq\left(\begin{array}{l}
x+1 \\
h+1
\end{array}\right)=\frac{x+1}{h+1} m, \\
\omega_{G}(h, h+1, m) & \leq m^{\langle h\rangle}
\end{aligned}
$$

respectively, where $x \geq h$ satisfies $\left(\begin{array}{l}x \\ h\end{array}\right)=m$. Applied to the case $(h, m)=$ $(5,100)$ in Section 4.1 , these bounds yield successively

$$
\omega_{G}(5,6,100) \leq \begin{cases}251 & \text { by } 123 \\ 161 & \text { by } \\ 152 & \text { by }\end{cases}
$$

The last one is probably close to optimal. Indeed, for $G=\mathbb{Z}$, we gave an example with $|5 A|=100$ and $|6 A|=145$, yielding $\omega_{\mathbb{Z}}(5,6,100) \geq 145$. Conjecture 4.7 implies that this is best possible, i.e. that $\omega_{\mathbb{Z}}(5,6,100)=145$. 
As another natural question, can one specialize Macaulay's theorem by characterizing the Hilbert functions of all algebras of the form $R(A)$ for finite subsets $A$ of a given abelian group $G$ ? A positive answer would help tackle the former question.

Finally, in a sequel to this paper, we will show two more aspects of the strength of Theorem 4.3. The proof methods are quite different from the present ones, except that Macaulay's theorem remains central. First, we will show that Theorem 4.3 is asymptotically optimal: the upper bound it provides, namely

$$
|(h+1) A| \leq|h A|^{\langle h\rangle}
$$

for all $h \geq 1$, is in fact an equality for $h$ large enough. Second, we will show that Theorem 4.3 is best possible in the sense that, given any sequence of positive integers $\left(d_{i}\right)_{i \geq 0}$ such that $d_{0}=1$ and

$$
1 \leq d_{i+1} \leq d_{i}^{\langle i\rangle}
$$

for all $i \geq 1$, there exists a finite subset $A$ of a commutative semigroup $(G,+)$ such that

$$
d_{h}=|h A|
$$

for all $h \geq 0$.

Together, the present paper and its forthcoming sequel raise the prospect that Macaulay's theorem, an almost century-old classical result from commutative algebra, may emerge as a powerful new tool in additive combinatorics.

Acknowledgments. This research was supported in part by the International Centre for Theoretical Sciences (ICTS) during a visit for the program - Workshop on Additive Combinatorics (Code: ICTS/wac2020/02). We are grateful to David Grynkiewicz for very useful discussions concerning this work during the ICTS Workshop.

\section{References}

[1] W. Bruns and J. Herzog, Cohen-Macaulay rings. Cambridge Studies in Advanced Mathematics, 39. Cambridge University Press, Cambridge, 1993. 
[2] S. Eliahou, Idéaux de définition des courbes monomiales. Complete intersections (Acireale, 1983), 229-240, Lecture Notes in Math., 1092, Springer, Berlin, 1984.

[3] S. Eliahou, Wilf's conjecture and Macaulay's theorem, J. Eur. Math. Soc. 20 (2018) 2105-2129.

[4] S. Eliahou and J. Fromentin, Near-misses in Wilf's conjecture, Semigroup Forum 98 (2019) 285-298.

[5] S. Eliahou and R.H. Villarreal, On systems of binomials in the ideal of a toric variety, Proc. Amer. Math. Soc. 130 (2002) 345-351.

[6] A.G. Khovanskit, Newton Polyhedron, Hilbert Polynomial, and Sums of Finite Sets, Funct. Anal. Appl. 26 (1992) 276-281.

[7] A.G. Khovanskil, Sums of Finite Sets, Orbits of Commutative Semigroups, and Hilbert Functions, Funct. Anal. Appl. 29 (1995) $102-112$.

[8] V.F. LEV, Structure theorem for multiple addition and the Frobenius problem, J. Number Theory 58 (1996) 79-88.

[9] F.S. Macaulay, Some properties of enumeration in the theory of modular systems, Proc. Lond. Math. Soc. 26 (1927) 531-555.

[10] J. Mermin And I. Peeva, Hilbert functions and lex ideals, J. Algebra 313 (2007) 642-656.

[11] M.B. Nathanson, Additive Number Theory, Inverse Problems and the Geometry of Sumsets. Graduate Texts in Mathematics, vol. 165, Springer, New York, 1996.

[12] M.B. Nathanson, Growth of sumsets in abelian semigroups, Semigroup Forum 61 (2000) 149-153.

[13] I. Peeva, Graded syzygies. Algebra and Applications, 14. SpringerVerlag London, Ltd., London, 2011.

[14] G. Petridis, The Plünnecke-Ruzsa inequality: an overview. In Combinatorial and additive number theory-CANT 2011 and 2012, 229241, Springer Proc. Math. Stat., 101, Springer, New York, 2014.

[15] H. PlünneCKe, Eine zahlentheoretische Anwendung der Graphentheorie, J. Reine Angew. Math. 243 (1970) 171-183.

[16] I.Z. RuzSA, Sumsets and structure. Combinatorial number theory and additive group theory, 87-210, Adv. Courses Math. CRM Barcelona, Birkhäuser Verlag, Basel, 2009. 
[17] I.Z. RuzSA, Arithmetical progressions and the number of sums, Period. Math. Hungar. 25 (1992), no. 1, 105-111.

[18] T. TaO And V. Vu, Additive combinatorics. Cambridge Studies in Advanced Mathematics, 105. Cambridge University Press, Cambridge, 2006. xviii+512 pp. ISBN: 978-0-521-85386-6; 0-521-85386-9.

[19] https://en.wikipedia.org/wiki/Binomial_coefficient.

[20] Wolfram Research, Inc., Mathematica, Version 10, Champaign, IL (2014).

\section{Authors' addresses:}

$\triangleright$ Shalom Eliahou, Univ. Littoral Côte d'Opale, UR 2597 - LMPA - Laboratoire de Mathématiques Pures et Appliquées Joseph Liouville, F-62228 Calais, France and CNRS, FR2037, France. eliahou@univ-littoral.fr

$\triangleright$ Eshita Mazumdar, Stat-Math Unit, ISI Bengaluru. eshita_vs(at)isibang.ac.in 